\title{
A rotary and reciprocating scintillator based fast-ion loss detector for the MAST-U tokamak
}

Cite as: Rev. Sci. Instrum. 89, 101112 (2018); https://doi.org/10.1063/1.5039311

Submitted: 07 May 2018 . Accepted: 11 June 2018. Published Online: 21 September 2018

J. F. Rivero-Rodriguez, M. Garcia-Munoz (D, R. Martin, J. Galdon-Quiroga, J. Ayllon-Guerola (D), R. J. Akers, J. Buchanan, D. Croft, D. Garcia-Vallejo (D), J. Gonzalez-Martin (D), D. Harvey (D, K. G. McClements (D), M. Rodriguez-Ramos, and L. Sanchis

\section{COLLECTIONS}

Paper published as part of the special topic on Proceedings of the 22nd Topical Conference on High-Temperature Plasma Diagnostics

Note: Paper published as part of the Proceedings of the 22nd Topical Conference on High-Temperature Plasma Diagnostics, San Diego, California, April 2018.
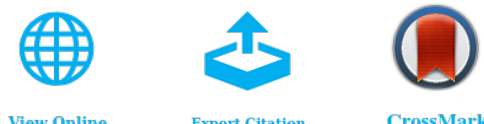

\section{ARTICLES YOU MAY BE INTERESTED IN}

First measurements of a scintillator based fast-ion loss detector near the ASDEX Upgrade divertor

Review of Scientific Instruments 89, 101106 (2018); https://doi.org/10.1063/1.5038968

The neutron camera upgrade for MAST Upgrade

Review of Scientific Instruments 89, 101110 (2018); https://doi.org/10.1063/1.5038948

A fast feedback controlled magnetic drive for the ASDEX Upgrade fast-ion loss detectors Review of Scientific Instruments 87, 11E705 (2016); https://doi.org/10.1063/1.4959913

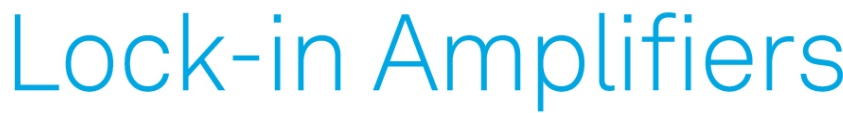
... and more, from DC to $600 \mathrm{MHz}$

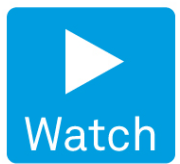




\title{
A rotary and reciprocating scintillator based fast-ion loss detector for the MAST-U tokamak
}

\author{
J. F. Rivero-Rodriguez, ${ }^{1,2, a)}$ M. Garcia-Munoz, ${ }^{2,3}$ R. Martin, ${ }^{4}$ J. Galdon-Quiroga, ${ }^{2,3}$ \\ J. Ayllon-Guerola, ${ }^{1,2}$ R. J. Akers, ${ }^{4}$ J. Buchanan, ${ }^{4}$ D. Croft, ${ }^{4}$ D. Garcia-Vallejo, ${ }^{1}$ \\ J. Gonzalez-Martin, ${ }^{1,2}$ D. Harvey, ${ }^{4}$ K. G. McClements, ${ }^{4}$ M. Rodriguez-Ramos, ${ }^{2,3}$ \\ and L. Sanchis ${ }^{2,3}$ \\ ${ }^{1}$ Department of Mechanical Engineering and Manufacturing, University of Seville, \\ Camino de los Descubrimientos s/n, 41092 Seville, Spain \\ ${ }^{2}$ Centro Nacional de Aceleradores (CNA), Universidad de Sevilla, CSIC, Junta de Andalucia, Seville, Spain \\ ${ }^{3}$ Department of Atomic, Molecular and Nuclear Physics, University of Seville, 41012 Seville, Spain \\ ${ }^{4}$ CCFE, Culham Science Centre, Abingdon, Oxon OX14 3DB, United Kingdom
}

(Presented 16 April 2018; received 7 May 2018; accepted 11 June 2018; published online 21 September 2018)

\begin{abstract}
The design and unique feature of the first fast-ion loss detector (FILD) for the Mega Amp Spherical Tokamak - Upgrade (MAST-U) is presented here. The MAST-U FILD head is mounted on an axially and angularly actuated mechanism that makes it possible to independently adapt the orientation $\left[0^{\circ}, 90^{\circ}\right]$ and radial position $[1.40 \mathrm{~m}, 1.60 \mathrm{~m}]$ of the FILD head, i.e., its collimator, thus maximizing the detector velocity-space coverage in a broad range of plasma scenarios with different $\mathrm{q}_{95}$. The 3D geometry of the detector has been optimized to detect fast-ion losses from the neutral beam injectors. Orbit simulations are used to calculate the strike map and predict the expected signals. The results show a velocity-space range of $[4 \mathrm{~cm}, 13 \mathrm{~cm}]$ in gyroradius and $\left[30^{\circ}, 85^{\circ}\right]$ in pitch angle, covering the entire neutral beam ion energy range. The optical system will provide direct sight of the scintillator and simultaneous detection with two cameras, giving high spatial and temporal resolution. The MAST-U FILD will shed light on the dominant fast-ion transport mechanisms in one of the world's two largest spherical tokamaks through absolute measurements of fast-ion losses. https://doi.org/10.1063/1.5039311
\end{abstract}

\section{INTRODUCTION}

Fast-ions are the main source of plasma heating in magnetically confined fusion devices. Energetic fusion-born $\alpha$ particles are needed to uphold a burning state. In present devices, fast-ions are mainly generated by external heating, such as Neutral Beam Injection (NBI) or Ion Cyclotron Resonant Heating (ICRH). Fast-ions also play a great role in non-inductive current drive, so they can be crucial in the realization of non-pulsed operation. Therefore, good fast-ion confinement is necessary to achieve high plasma temperatures and non-inductive current drive. Additionally, fast-ion losses may affect the device integrity by severely damaging plasma facing components. ${ }^{1}$

The Fast-Ion Loss Detector (FILD) $)^{2,3}$ is a unique diagnostic in the detection of MHD induced fast-ion losses. ${ }^{4}$ It works as a magnetic spectrometer, inferring the escaping fastion velocity-space by their strike point on a scintillator plate, as illustrated in Fig. 1. They have been used in most large tokamaks, such as TFTR, ${ }^{2,5}$ ASDEX Upgrade, ${ }^{3,6}$ JET, ${ }^{7}$ NSTX, ${ }^{8}$ and DIII-D, ${ }^{9}$ among others.

In this work, the first FILD for the Mega Amp Spherical Tokamak - Upgrade (MAST-U) has been designed and implemented, as part of the major upgrade that this spherical

Note: Paper published as part of the Proceedings of the 22nd Topical Conference on High-Temperature Plasma Diagnostics, San Diego, California, April 2018.

a)Electronic mail: jfrivero@us.es tokamak is currently undergoing. ${ }^{10}$ The MAST-U FILD incorporates the ability to rotate its head in a shot-to-shot basis, thus adapting its aperture orientation to the plasma $\mathrm{q}_{95}$. This is a unique feature for any FILD system, and it is especially important in a spherical tokamak, where the broader range of $\mathrm{q}_{95}$ will make it necessary to reorientate the FILD aperture in order to preserve acceptable velocity-space coverage.

This paper is structured as follows. The FILD working principle is presented in Sec. I. The probe head design using the FILDSIM code is shown in Sec. II. The distinctive features of the rotary and reciprocating system are presented in Sec. III, as well as a finite element analysis (FEA) of the in-vessel system. An outline of the data acquisition system is given in Sec. IV. Section V summarises the current status and outlook of this diagnostic.

\section{FILD PROBE DESIGN}

The fast-ion population that FILD aims to measure is that produced by the NBI heating. Two NBIs (on-axis and off-axis) will inject deuterium (or hydrogen) at a maximum energy of $75 \mathrm{keV}$. The half $(37.5 \mathrm{keV})$ and third $(25 \mathrm{keV})$ injection energies should also be considered. The local magnetic field on the FILD probe will be in the range of $B=[0.45 \mathrm{~T}, 0.55 \mathrm{~T}]$. Thus, the fast-ion gyroradius will be in the range of $\rho=[41 \mathrm{~mm}$, $125 \mathrm{~mm}]$.

The FILD probe head is designed making use of the FILDSIM code. ${ }^{11}$ FILDSIM takes into account the probe head 
a)
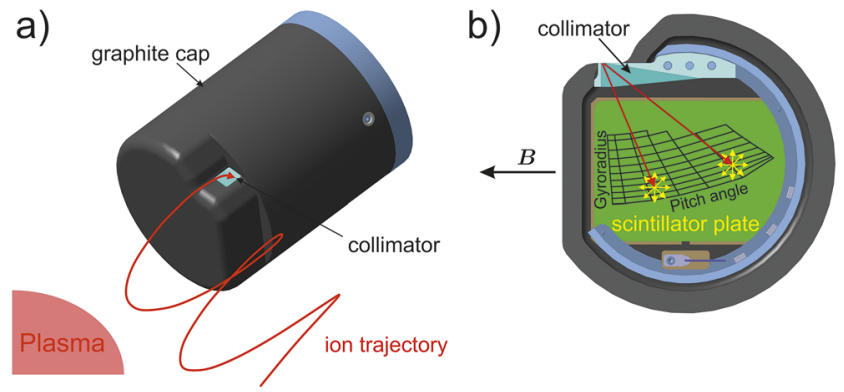

FIG. 1. (a) FILD probe head and escaping fast-ion orbit reaching the aperture. (b) Scintillator plate where the collimated ions are dispersed using the tokamak magnetic field and characterized by their gyroradius and pitch angle.

3D geometry and uses orbits forward tracing from the pinhole to produce a synthetic FILD signal. With this, one can estimate the following:

- Strike map

- Collimator factor

- Velocity-space coverage

- Signal resolution.

Besides, FILDSIM carries out backward tracing to estimate the velocity-space range that would be self-obstructed by the probe head external geometry.

The strike map for the final collimator geometry is shown in Fig. 2(a). Moreover, the synthetic signal of deuterium
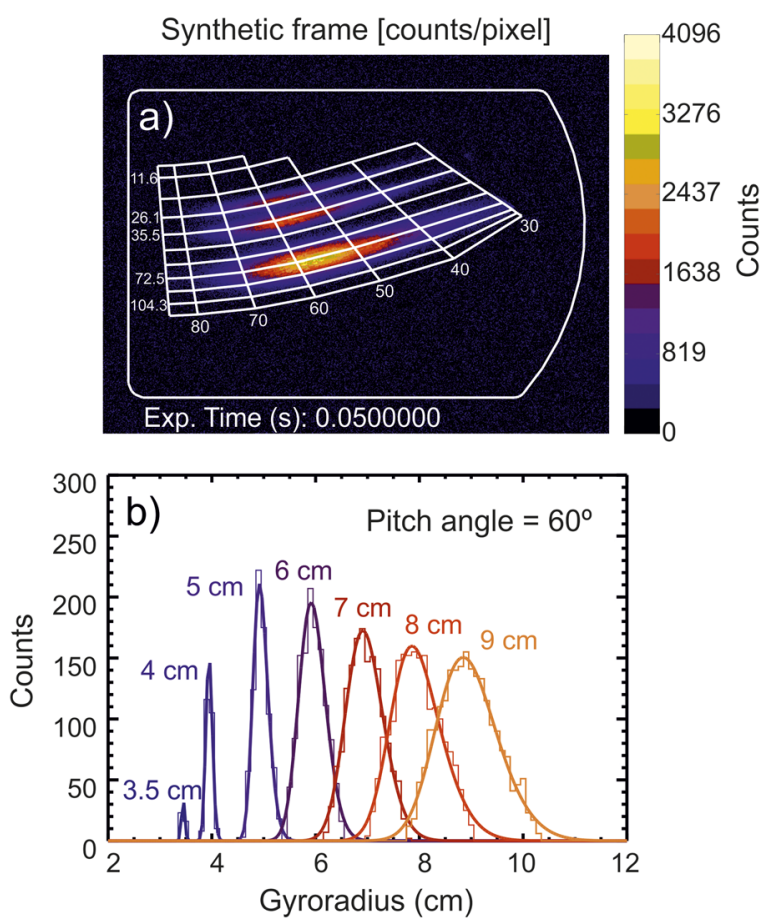

FIG. 2. (a) Synthetic signal for an artificial distribution of deuterium at the main $(E=75 \mathrm{keV})$, half $(E=37.5 \mathrm{keV})$, and third $(E=25 \mathrm{keV}) \mathrm{NBI}$ injection energies, corresponding to $\rho=10.1 \mathrm{~cm}, \rho=7.2 \mathrm{~cm}$, and $\rho=5.8 \mathrm{~cm}$ gyroradius values, respectively, for a $B=0.55 \mathrm{~T}$ magnetic field. The distribution covers the entire pitch angle range of the FILD probe. The overlaid strike map shows the energy ( $\mathrm{keV})$ on the vertical axis and the pitch angle values $\left({ }^{\circ}\right)$ on the horizontal axis, assuming $B=0.55 \mathrm{~T}$ and deuterium as the impinging ion species. (b) Energy dispersion on the scintillator plate for several mono-energetic distributions on the pinhole. at $75 \mathrm{keV}, 37.5 \mathrm{keV}$, and $25 \mathrm{keV}$ is also shown, showing that the collimator geometry delivers enough energy resolution to separate the three NBI energy components of injected deuterium. This geometry stops gyroradii below $\rho=35 \mathrm{~mm}$, thus avoiding thermal ion detection. It is worth mentioning that a larger Larmor radius than the maximum shown in the strike map would not be obstructed by the collimator and thus fast-ions at higher energies could in principle be measured, with the range only limited by the scintillator plate size. Backward tracing shows that the external geometry severely obstructs orbits below $\rho=40 \mathrm{~mm}$, thus not affecting the FILD signal.

Figure 2(b) shows how the detector resolution in gyroradius lessens at larger gyroradius values. This is geometrically caused by the collimator finite dimensions and is difficult to avoid. $^{3}$ On the contrary, the resolution in pitch angle remains almost constant for the entire velocity-space range, ${ }^{11}$ being $\Lambda= \pm 2^{\circ}$. The overall detection resolution can be enhanced using tomographic reconstruction in the analysis of the FILD signal, using the FILDSIM code.

\section{ROTARY AND RECIPROCATING SYSTEM}

In MAST-U, the FILD probe head is mounted on an axially and angularly actuated mechanism. This means that the probe head position can be modified by two independent settings on a shot-to-shot basis, as it is illustrated in Fig. 3(a). One setting makes it possible to modify the probe head radial position in a range of $R=[1.40 \mathrm{~m}, 1.60 \mathrm{~m}]$, adapting its distance from the plasma similar to the FILD systems in ASDEX Upgrade and DIII-D. The other setting enables changes in the aperture orientation in a range of $90^{\circ}$. It will make possible to adapt the aperture orientation to plasma scenarios with different $q_{95}$ without losing velocity-space coverage. This is an especially relevant feature for a spherical tokamak, where the use of lower toroidal fields leads to a broader range of plasma q95. Thus, MAST-U FILD has unique versatility in terms of being capable of adapting to different plasma scenarios, maximizing the detector velocity-space coverage.

The mechanical system is shown in Fig. 3(b). Since the MAST-U FILD probe head only allows for measurements in forward field experiments ( $B_{t}$ clockwise), the detector is located $109 \mathrm{~mm}$ above the midplane, where the fast-ion loss detection will be enhanced by the grad-B drift, pointing upward. Its modular telescopic design results in a compact system for an otherwise large detector. Light-tight parts enable direct sight to the scintillator plate, placing the optical system outside the vacuum vessel. In-vessel high friction contacts have been avoided and special care has been taken in the material selection of contact bodies to prevent cold welding. The vacuum boundary consists of the port plate and bellow-based drives, ensuring its integrity. The graphite cap design has been revised to allow free thermal expansion, and its temperature will be monitored by a thermocouple during operation. In summary, the mechanical system provides accurate positioning of the detector head while prioritising the detector integrity and safety.

The in-vessel assembly is bolted to a port plate, forming a cantilever of about $30 \mathrm{~kg}$ supported by it. Therefore, good structural performance of FILD is required to guarantee the 

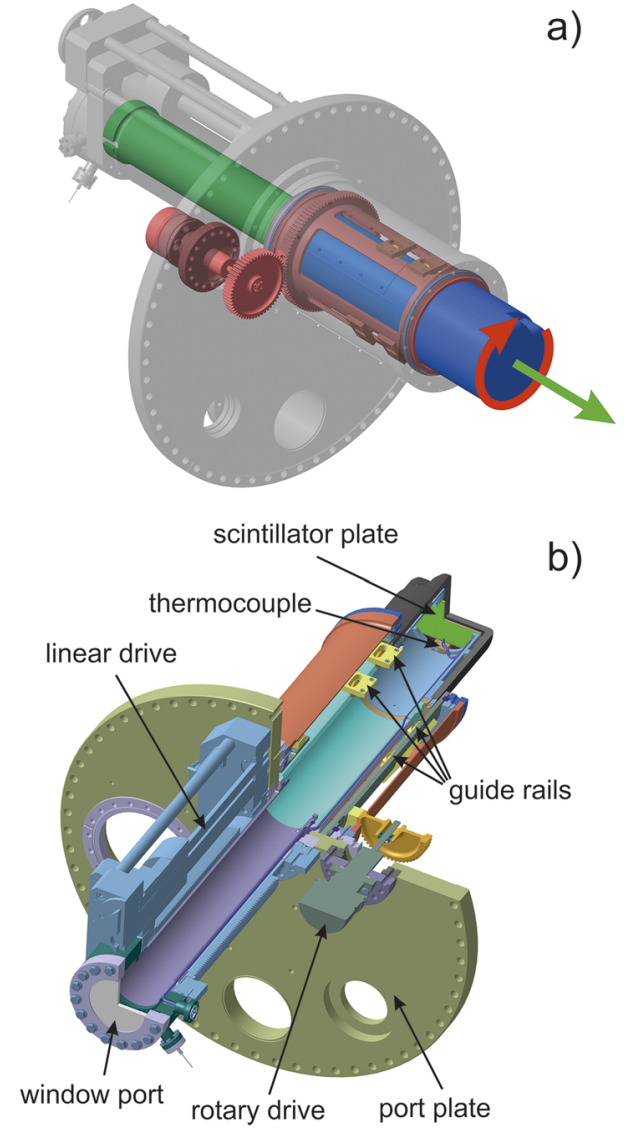

FIG. 3. (a) Rotary (red) and reciprocating (green) system that drives the FILD probe head (blue). (b) Overview of the MAST-U FILD mechanical system.

port plate integrity. In order to assess this, a finite element analysis (FEA) of the in-vessel structure has been carried out in ANSYS ${ }^{\circledR}$ workbench. The analysis mimics a shock caused by

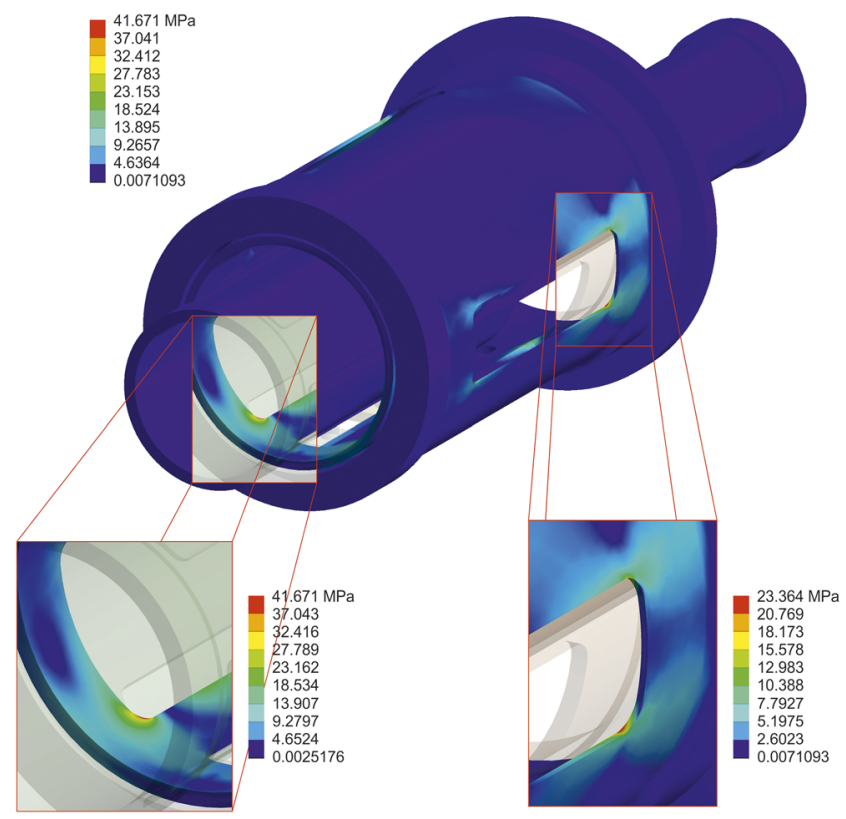

FIG. 4. Equivalent von Mises stress on the in-vessel structural components. Stress concentration areas are highlighted. a disruption simulating a $2 \mathrm{kA}$ current going through the FILD structure. Figure 4 shows the resulting von Mises stress on the system. The highlighted stress concentration areas undergo stress magnitudes 5 times below the material yield stress. The force reactions on the rotary bearings are 6 times below the maximum recommended by the manufacturer. Therefore, the FEA foresees good structural performance of the in-vessel system. The FEA also shows that the guide rails supporting the probe head [shown in Fig. 3(b)] will be the most critical parts of the system, so its performance will be monitored during the detector operation.

\section{LIGHT ACQUISITION SYSTEM}

The scintillator plate is visible from a window port at the rear end of the mechanical system. This makes it possible to place the optical system and data acquisition system outside the vacuum vessel, providing full accessibility during experimental campaigns.

The optical system is shown in Fig. 5, and each optical element is described in Table I. It is formed exclusively by lenses, leveraging the scintillator plate full resolution, in contrast to systems where the use of fibre optics limits the signal resolution to the size of the fibre bundle. A beam splitter allows monitoring of the scintillator plate by two different cameras simultaneously. One camera provides good spatial resolution to capture sharp pictures of the scintillator plate. With this, one can resolve the velocity-space of the impinging ions using the strike map described in Sec. II. The other camera is an avalanche photo-diode (APDCAM 10G) ${ }^{12}$ camera with a sampling rate of $2 \mathrm{MHz}$. This in combination with a fast scintillator material (P46) makes it possible to detect fast-ion loss fluctuations, which can be correlated with the MHD activity of the plasma.

Due to its high sampling frequency, the APD camera has relatively high memory consumption, requiring up to $2.5 \mathrm{~GB}$ per shot. For this reason, a dedicated personal computer (PC) with enough memory is required to acquire and control the

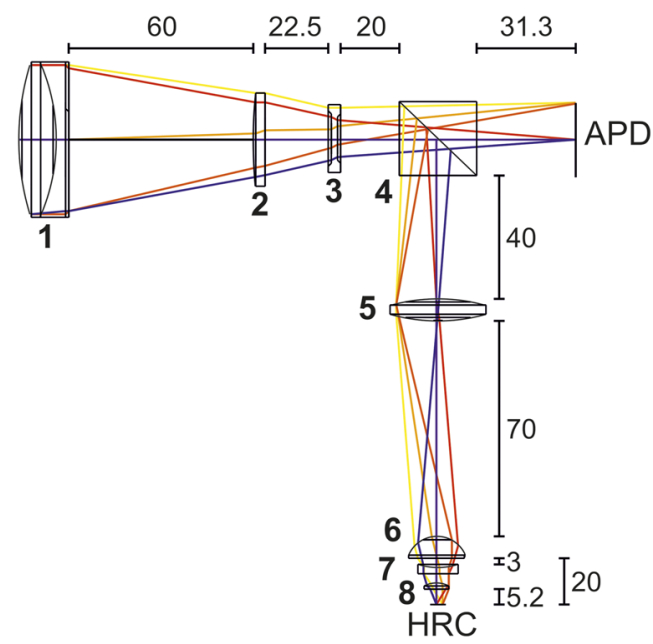

FIG. 5. Optical system and ray tracing to the APD camera and the high resolution camera. The distance between elements (in $\mathrm{mm}$ ) and the numeration corresponding to Table I are also shown. 
TABLE I. Type, focal length, and diameter of each optical element, according to the numeration set in Fig. 5.

\begin{tabular}{llcc}
\hline \hline Element & \multicolumn{1}{c}{ Type } & Focal length $(\mathrm{mm})$ & $\emptyset(\mathrm{mm})$ \\
\hline 1 & Achromat & 120 & 50 \\
2 & Plano-convex & 300 & 31.5 \\
3 & Symmetric-concave & -50 & 22.4 \\
4 & Beam splitter & & \\
5 & Symmetric-convex & 50 & 31.5 \\
6 & Aspheric & 15 & 18 \\
7 & Plano-concave & -30 & 12.7 \\
8 & Plano-convex & 12.5 & 8 \\
\hline \hline
\end{tabular}

APD data. However, the high resolution camera is not as demanding, requiring about $100 \mathrm{MB}$ per shot. Thus, a second PC will be used to control both the high resolution camera and the motors that drive the probe head linear and rotary motions.

\section{SUMMARY}

The first MAST-U FILD, shown in Fig. 6, is now designed and manufactured. It provides enough velocity-space coverage and resolution to diagnose the NBI fast-ion population in a broad range of plasma scenarios. The mechanical system includes the ability to adapt the FILD aperture orientation, a unique feature in any FILD device. This is combined with a reciprocating system in a compact mechanism that provides the detector with unprecedented adaptability to plasma shapes.

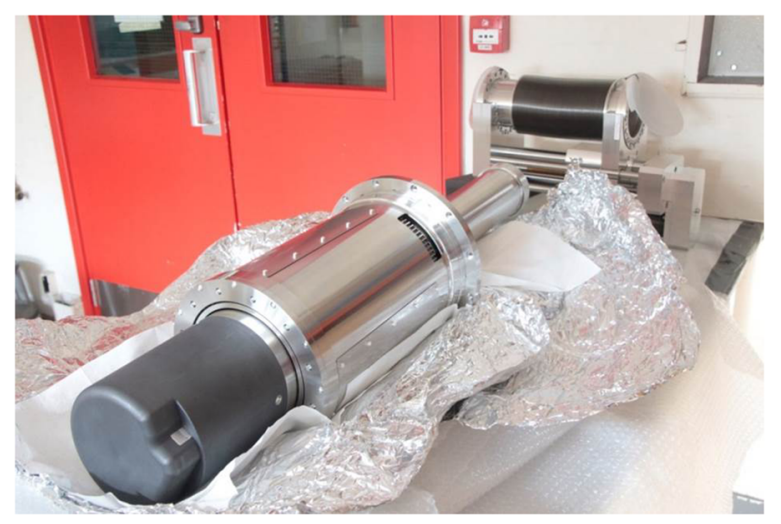

FIG. 6. Picture of the assembled MAST-U FILD.
The diagnostic is to be installed in MAST-U. Then, the commissioning will be carried out in parallel to the commissioning of other MAST-U systems and its conclusion will require the operation of the NBI heating. Eventually, FILD will be into service from the first MAST-U campaign, planned for January 2019. To that end, fast-ion modeling is ongoing to guide the fast-ion experiments.

\section{ACKNOWLEDGMENTS}

This work has been carried out within the framework of the EUROfusion Consortium and has received funding from the Euratom research and training programme 2014-2018 under Grant Agreement No. 633053. The views and opinions expressed herein do not necessarily reflect those of the European Commission. This research received funding from the V Plan Propio de Investigacion de la Universidad de Sevilla (PP2016-7145).

The authors would like to thank the MAST Upgrade team for their help with the technical aspects of this project.

${ }^{1}$ J. Galdon-Quiroga, M. Garcia-Munoz, L. Sanchis-Sanchez, M. Mantsinen, S. Fietz, V. Igochine, M. Maraschek, M. Rodriguez-Ramos, B. Sieglin, A. Snicker, G. Tardini, D. Vezinet, M. Weiland, and L. Eriksson, Nucl. Fusion 58, 036005 (2018).

${ }^{2}$ S. Zweben, Nucl. Fusion 29, 825 (1989).

${ }^{3}$ M. García-Muñoz, H. U. Fahrbach, and H. Zohm, Rev. Sci. Instrum. 80, 053503 (2009).

${ }^{4}$ M. García-Muñoz, H. U. Fahrbach, S. Günter, V. Igochine, M. J. Mantsinen, M. Maraschek, P. Martin, P. Piovesan, K. Sassenberg, and H. Zohm, Phys. Rev. Lett. 100, 055005 (2008).

${ }^{5}$ S. J. Zweben, R. L. Boivin, C.-S. Chang, M. Diesso, S. Hayes, H. W. Hendel, H. Park, and J. D. Strachan, Nucl. Fusion 30, 1551 (1990).

${ }^{6}$ J. Ayllon-Guerola, J. Gonzalez-Martin, M. Garcia-Munoz, J. RiveroRodriguez, A. Herrmann, S. Vorbrugg, P. Leitenstern, S. Zoletnik, J. Galdon, J. Garcia Lopez, M. Rodriguez-Ramos, L. Sanchis-Sanchez, A. D. Dominguez, M. Kocan, J. P. Gunn, D. Garcia-Vallejo, and J. Dominguez, Rev. Sci. Instrum. 87, 11E705 (2016).

${ }^{7}$ S. Baeumel, A. Werner, R. Semler, S. Mukherjee, D. S. Darrow, R. Ellis, F. E. Cecil, L. Pedrick, H. Altmann, V. Kiptily, and J. Gafert, Rev. Sci. Instrum. 75, 3563 (2004).

${ }^{8}$ D. S. Darrow, Rev. Sci. Instrum. 79, 023502 (2008).

${ }^{9}$ R. K. Fisher, D. C. Pace, M. Garca-Munoz, W. W. Heidbrink, C. M. Muscatello, M. A. V. Zeeland, and Y. B. Zhu, Rev. Sci. Instrum. 81, 10 D307 (2010).

${ }^{10}$ A. W. Morris, IEEE Trans. Plasma Sci. 40, 682 (2012).

${ }^{11}$ J. Galdon-Quiroga, M. Garcia-Munoz, M. Salewski, A. S. Jacobsen, L. Sanchis-Sanchez, M. Rodriguez-Ramos, J. Ayllon-Guerola, J. GarciaLopez, J. Gonzalez-Martin, M. C. Jimenez-Ramos, J. F. Rivero-Rodriguez, and E. Viezzer, Plasma Phys. Controlled Fusion 60, 105005 (2018).

${ }^{12}$ D. Dunai, S. Zoletnik, J. Sárközi, and A. R. Field, Rev. Sci. Instrum. 81, 103503 (2010). 